\begin{tabular}{|c|l|}
\hline Title & Surfactant-Induced Friction Reduction for Hydrogels in the Boundary Lubrication Regime \\
\hline Author(s) & $\begin{array}{l}\text { Kamada, Kosuke; Furukawa, Hidemitsu; Kurokawa, Takay uki; Tada, Tomohiro; Tominaga, Taiki; Nakano, Y ukihiro; } \\
\text { Gong, Jian Ping }\end{array}$ \\
\hline Citation & $\begin{array}{l}\text { Journal of Physics: Condensed Matter, 23, 284107 } \\
\text { https://doi.org/L0.1088/0953 8984/23/28/284107 }\end{array}$ \\
\hline Issue Date & 2010-11-26 \\
\hline Doc URL & http://hdl.handle.net/2115/44831 \\
\hline Rights & Copyright @ (2011) IOP Publishing Ltd. \\
\hline Type & article (author version) \\
\hline File Information & kamadaT EX 101027-revised 101112.pdf \\
\hline
\end{tabular}

Instructions for use 


\title{
Surfactant-Induced Friction Reduction for Hydrogels in Boundary Lubrication Regime
}

Kosuke Kamada ${ }^{1}$, Hidemitsu Furukawa ${ }^{2,3}$, Takayuki Kurokawa ${ }^{2,4}$, Tomohiro Tada ${ }^{l}$, Taiki Tominaga ${ }^{l}$, Yukihiro Nakano ${ }^{1}$, and Jian Ping Gong ${ }^{2, *}$

${ }^{1}$ Graduate School of Science, Hokkaido University, Sapporo 060-0810, Japan

${ }^{2}$ Faculty of Advanced Life Science, Hokkaido University, Sapporo 060-0810, Japan

${ }^{3}$ Department of Mechanical Systems Engineering, Graduate School of Science and Engineering, Yamagata University, Yamagata 992-8510, Japan

${ }^{4}$ Creative Research Initiative Sousei, Hokkaido University, Sapporo 001-0021, Japan

*Corresponding author. E-mail: gong@sci.hokudai.ac.jp.

\begin{abstract}
We studied the ability of surfactants to reduce friction by boundary lubrication for a bulk hydrogel sliding on a solid surface in aqueous solution. A piece of negatively charged polyelectrolyte hydrogel was slid across solid surfaces with various levels of hydrophobicity, using a strain-controlled parallel-plate rheometer in water. A dramatic reduction in the sliding friction, especially in the lowvelocity region, was detected by the addition of a surfactant to the water medium. This friction reduction was only observed in gel-solid friction but not in solid-solid friction, indicating that the soft and wet nature of the gel surface was crucial for this surfactant-induced friction reduction. This phenomenon reveals that surfactants can remain at the gel-mated interface, thus preventing direct interfacial interaction between the sliding surfaces, and significantly decreases the frictional stress. The reported dramatic reduction in friction highlights the frictional characteristics of soft and wet hydrogel materials.
\end{abstract}




\section{Introduction}

A hydrogel, contains $60-99.9 \mathrm{wt} \%$ water in a cross-linked hydrophilic polymer network, belongs to a class of soft and wet material. Depending on its water content, a hydrogel exhibits an elastic modulus in the range of 1-1000 kPa, covering the whole elastic range of the biological soft tissues [1]. Studying on the surface sliding friction of hydrogel in aqueous solution has recently been drawing great scientific [2-10] and engineering [11-15] attentions. One reason is that hydrogel friction study is important to understand the fascinating low friction phenomenon of the biological soft tissues that are also belong to the soft and wet material, such as the friction between an eye and an eyelid, the friction between an endothelial cell (EC) and a red blood cell, and the friction between articular cartilages in human joints, etc. $[16,17]$. Understanding of biological lubrication has now advanced to the point where these principles can be applied to systems of technological importance using synthetic polymers[18].

Previous studies have shown that hydrogels exhibit complex but unique frictional behaviors [2-5]. For example, they have a wide range of frictional coefficients in water against a smooth solid, ranging from $10^{-3}$ to $10^{0}$ in magnitude, depending on various parameters such as the interfacial interaction between the polymer network and the solid, the normal pressure, and the sliding velocity [2]. A recent study on the friction between two like-charged polyelectrolyte gels in pure water showed that the frictional behavior is also dependent on the elasticity of the gel and its thickness [19]. The frictional stress demonstrates strong velocity dependence (liquid-like) when the gel is soft and thick, but demonstrates weak or even no velocity dependence (solid-like) when the gel is rigid and thin. The former is interpreted as the combined mechanisms of boundary lubrication and hydrated lubrication, wherein the boundary lubrication is due to the direct contact of polymer network to the substrate and the hydrated lubrication is due to the formation of a velocity-independent electric double layer at the soft and repulsive interfaces. On the other hand, the latter is interpreted as the combined mechanisms of boundary lubrication and elastohydrodynamic lubrication, wherein the elastohydrodynamic lubrication is due to the velocity-enhanced water entrainment that occurs during sliding. In either cases, the frictional stress $\sigma$ can be analyzed phenomenologically by considering two components: a velocityindependent component $\sigma_{0}$ in the boundary lubrication regime, where the highly compressed asperities 
come into contact; and a velocity-dependent component $\sigma_{\text {vis }}$ as a result of the viscous energy dissipation, i. e., lubrication, by the water layer that exists interstitially at the repulsive interface [19]; that is,

$$
\sigma=\sigma_{0}+\sigma_{\text {vis }}
$$

$\sigma_{\mathrm{vis}}=\eta v / h$, where $\eta$ is the viscosity of water, $v$ is the sliding velocity, and $h$ is the apparent thickness of the liquid film formed at the interface [19]. The boundary lubrication component $\sigma_{0}$, which is the predominant term at low velocity regime, is strongly dependent on the interfacial contact and interaction.

In this study, we attempted to reduce the frictional stress of the hydrogel that originated from the boundary lubrication component by using an approach similar to biological system. Effects of surfactant on the lubrication of hydrated soft biological surfaces are frequently observed. For example, beside in the eyes and in the cartilage of articular joints, surfactants are also been found to play a role as lubricant in the boundary lubrication of pleurae[20], the gastrointestinal tract[21], and peritoneal mesothelium [22]. Inspired from these biological results, we study the friction of hydrogel in various surfactant solutions.

We use the following system: We chose the chemically cross-linked poly(2-acrylamido-2methylpropanesulfonic acid sodium salt) (PNaAMPS) as the negatively charged gel. In order to highlight the frictional reduction effect of a surfactant, we chose a "rigid and thin" gel sample (elastic modulus, $0.8 \mathrm{MPa}$; thickness, $2.75 \mathrm{~mm}$ ), for which the boundary lubrication component $\sigma_{0}$ was predominant over the whole velocity range in pure water, according to the literature [19]. Furthermore, we chose negatively charged surfactant molecules to avoid the unnecessary adsorption of the surfactant molecules on the gel, which might have caused large changes in the degree of swelling, sample size, and elastic modulus of the gel in the surfactant solutions. Furthermore, we chose the following as the mating substrates for the friction test: hydrophilic glass, hydrophobically treated glass (D-glass), and silicon wafers. These substrates allowed us to investigate the effect of the surfactant on lubrication as the interfacial interaction between the gel and the mating surface changed. 
This paper consists of three sections. After this section, we present the experimental procedures in Section II. In Section III, we present the frictional behavior of PNaAMPS gel as it slides against hard solid substrates with various levels of hydrophobicity in media consisting of anionic surfactant solutions, and we discuss experimental observations on the basis of the combined mechanisms of boundary and hydrodynamic lubrication. In Section IV, we present our conclusions.

\section{Experimental Section}

\section{Materials}

2-Acrylamido-2-methylpropanesulfonic acid (AMPS) (Tokyo Kasei Co., Ltd.) was used as received and neutralized with sodium hydroxide (Junsei Chemical Co., Ltd.) to obtain the sodium salt (NaAMPS). N,N'-Methylene bisacrylamide (MBAA) (Tokyo Kasei Co., Ltd.), used as the cross-linking agent, was recrystallized from ethanol. 2-Oxoglutaric acid (Wako Junyaku Co., Ltd.), used as a UVactivated radical initiator, was used without purification. Surfactants, sodium dodecyl sulfate (SDS) (Wako Junyaku Co., Ltd.), lauric acid (sodium salt; LASS; Tokyo Kasei Co., Ltd.), dodecylbenzene sulfonic acid (sodium salt; DBS; Tokyo Kasei Co., Ltd.), were used as received. Water was filtrated using a Millipore filter (Elix, Nihon Millipore Co., Ltd).

\section{Sample Preparation}

PNaAMPS gel was prepared by radical polymerization of a $1 \mathrm{M}$ aqueous solution of NaAMPS monomer in the presence of $6 \mathrm{~mol} \% \mathrm{MBAA}$. The solution was bubbled with argon gas for $30 \mathrm{~min}$. Next, $0.1 \mathrm{~mol} \%$ 2-oxoglutaric acid, an initiator, was added to the solution, which was then irradiated with UV light for $6 \mathrm{~h}$ at room temperature. The reaction was carried out between two parallel glass substrates separated by a $1.5-\mathrm{mm}$ spacer. After polymerization, the gel was immersed in a large amount of water for 1 week, after which the gel was immersed in a surfactant solution for 1 week. The chemical structures of the various ionic surfactant molecules used in the present study are shown in Table 1.

Three kinds of hard solid substrates were used, hydrophilic glass, silicon wafer, and glass with its surface treated by hydrophobic dansyl chloride solution (D-glass). The contact angles of water on these 
substrates were $25^{\circ}, 58^{\circ}$, and $85^{\circ}$ for hydrophilic glass, silicon wafer, and D-glass, respectively. The surface roughnesses of these surfaces were $2 \mathrm{~nm}, 0.9 \mathrm{~nm}$, and $5 \mathrm{~nm}$ for the hydrophilic glass, silicon wafer, and the D-glass, respectively. Due to the formation of the electric double layer, we could not measure the surface roughness of the PNaAMPS gel in pure water using AFM. However, for a neutral hydrogel synthesized on silicon substrate $(0.9 \mathrm{~nm}$ in roughness), the surface roughness of the gel was found to be $5 \mathrm{~nm}$ over a scanning area of $20 \times 20 \mu \mathrm{m}^{2}[23]$. Since the PNaAMPS gel has a higher swelling degree than the neutral hydrogel, the surface roughness of the PNaAMPS gel synthesized on the hydrophilic glass substrate ( $2 \mathrm{~nm}$ in roughness) should be much higher than $5 \mathrm{~nm}$.

\section{Friction Measurement}

A commercially available rheometer called ARES (Advanced Rheometric Expansion System, Rheometric Scientific, Inc.) was used for measuring the frictional stress. Sample gels at equilibrium in the swollen state in water or in various surfactant solutions were cut into disks (radius $R=7.5 \mathrm{~mm}$ ) using a cylindrical gel-cutter. The thickness, elasticity, and swelling degree of the gel swollen in water were $2.75 \pm 0.25 \mathrm{~mm}, 0.8 \mathrm{MPa}$, and 26 , respectively. Gels swollen in surfactant solutions exhibited the same thickness as that in water, indicating that their elasticity and swelling degree did not changes upon immersing in surfactant solutions. A disc-shaped glass sample with a radius of $12.5 \mathrm{~mm}$ and a thickness of $3 \mathrm{~mm}$ was also used as a reference. The sample was then glued to the upper surface of a coaxial disc-shaped plate using a cyanoacrylate-based instant adhesive (Toagosei Co., Ltd.). Hydrophilic glass, D-glass, and silicon wafers were used as the mating substrates. These substrate plates were much larger than the gel samples. During the measurement, each sample-substrate plate was immersed in pure water or the surfactant solution. The two surfaces were pressed together under a normal load of $0.4 \mathrm{~N}$ to attain a nominal pressure of $2.2 \mathrm{kPa}$. The substrate plate was rotated at an angular velocity $\omega$ that varied from $10^{-3}$ to $10^{0} \mathrm{rad} / \mathrm{s}$, corresponding to an average sliding velocity $V$ of $3.75 \times 10^{-6}$ to $3.75 \times 10^{-3} \mathrm{~m} / \mathrm{s}$ for the sample gels and $6.25 \times 10^{-6}$ to $6.25 \times 10^{-3} \mathrm{~m} / \mathrm{s}$ for the glass samples at a distance of $(1 / 2) R$ from the center of the sample. The torque of the rheometer was measured, and the shear stress (frictional stress) corresponding to the sliding velocity was $\sigma(\omega R)=[(3+\alpha) T(\omega)] /\left(2 \pi R^{3}\right)$, supposing that the torque has a power relation with $\omega$ of $T(\omega) \sim \omega^{\alpha}$. 
Except for the case that a sharp transition from boundary lubrication to hydrodynamic lubrication occurs, which was not the case of this study, $\alpha$ lies between 0 and 1 [2,5]. As an approximation, we simply calculated the frictional stress by setting $\alpha=1$. The frictional coefficient $\mu$ was calculated by dividing the frictional stress with the normal pressure. The details of this friction measurement method have been described in previous papers [5]. The friction of glass vs. glass was also measured in the same manner.

\section{Results}

We first investigated the friction of a negatively charged PNaAMPS gel on a glass substrate that was also negatively charged in an aqueous solution of $\mathrm{pH}$ 7. Figure 1(a) shows the sliding velocity dependence of the frictional stress $\sigma$ for the PNaAMPS gel sliding on glass in water and in an SDS solution of various concentrations $C_{\text {surf }}$. The $\sigma$ in water showed a slight velocity-weakening over a wide velocity range, indicating that the gel friction was dominated by the boundary lubrication, that is, the first term of Eq. 1, in agreement with the results in the previous observation [19]. In the low velocity region below $3.75 \times 10^{-4} \mathrm{~m} / \mathrm{s}$, the value of $\sigma$ in the SDS solutions rapidly decreased as the SDS concentration increased, especially at the lowest velocity limit. However, this surfactant effect became less prominent with an increase in the velocity, regardless of the change in the SDS concentration. The values of $\sigma$ in SDS solutions were quite close to, but even slightly higher than, that of water at the highest velocity, $3.75 \times 10^{-3} \mathrm{~m} / \mathrm{s}$. Since SDS is an ionic molecule that may also play a role as electrolyte in screening the electrostatic interaction between the charged gel and glass, we also studied the effect of ionic strength on the friction of this polyelectrolyte gel by measure the friction in $\mathrm{NaCl}$ solution. As shown by the solid circles in Figure 1(a), over the whole velocity range, the values of $\sigma$ in the $0.1 \mathrm{M} \mathrm{NaCl}$ solution were higher than those in water, which is opposite to the effect of SDS. This means that the reduction in friction was not due to the ionic strength effect of the SDS, but due to the surfactant nature of the SDS.

Similar friction reduction effect was observed for two other surfactants, LASS and DBS, when their concentration was high. However, when the surfactant concentration was low, the behaviors were different for these three surfactants, as shown in Figure 1(b) for LASS and Figure 1(c) for DBS. 
In order to elucidate whether this surfactant effect also exists for substrates with different hydrophobic levels, two additional substrates, silicon wafer and a glass substrate treated with dansyl chloride (D-glass), were used. Figure 2 shows the frictional stress $\sigma$ on the silicon wafer (Figure 2a) and D-glass (Figure 2b) in an SDS solution as a function of the sliding velocity. In water $\left(C_{\text {surf }}=0\right)$, the values of $\sigma$ on these substrates showed a velocity weakening behavior and the values of $\sigma$ at the low velocity limit were higher than that on the hydrophilic glass substrate. This indicates that the polymer chains on the surface of the PNaAMPS gel were strongly adsorbed on these substrates, especially on the D-glass, to produce a high frictional resistance, due to the hydrophobicity of the substrates. $\sigma$ decreased as $C_{\text {surf }}$ increased on all of the substrates, especially at the low sliding velocity, which was similar to the behavior on the hydrophilic glass substrate.

To investigate the relation between the friction reduction and the critical micelle concentration, $C_{\mathrm{CMC}}$ of the surfactants, we plotted $\sigma$ as a function of $C_{\text {surf }}$ for the PNaAMPS gel sliding on various substrates, at the low velocity limit of $3.75 \times 10^{-6} \mathrm{~m} / \mathrm{s}$. Figure 3 a shows the results for $\mathrm{SDS}$. In water $\left(\mathrm{C}_{\text {surf }}=0\right)$, the frictional stress depended on the hydrophobicity, that is, $\sigma$ followed the order of D-glass $>$ silicon $>$ glass. As $C_{\text {surf }}$ increased in the lower concentration region, $\sigma$ gradually decreased. However, as $C_{\text {surf }}$ increased further, $\sigma$ decreased dramatically until it reached a critical $C_{\text {surf }}=10^{-2} \mathrm{M}$. This critical concentration was very close to the $C_{\mathrm{CMC}}(=0.008 \mathrm{M})$ of SDS. A further increase in $C_{\text {surf }}$ brought about a modest change in $\sigma$. When $C_{\text {surf }}=0.3 \mathrm{M}$, about 30 times the value of $C_{\mathrm{CMC}}$, all of the $\sigma$ values were almost the same, regardless of the difference in the substrates. Based on the above results, we argue that $\sigma$ decreases regardless of the hydrophilic/hydrophobic property of the frictional substrate, due to the adsorption of SDS on the substrates, which screens the interaction between the polymer chain and the substrate. The adsorption of SDS increases with an increase in $C_{\text {surf, }}$ and probably forms a continuous layer around $C_{\text {surf }}=C_{\mathrm{CMC}}$, allowing us to observe the saturation of $\sigma$. A further increase in $C_{\text {surf }}$ may not change the interaction between the gel and the substrate, so that the values of $\sigma$ for different substrates merge into the same value as $C_{\text {surf }}$ increases to 30 times that of $C_{\mathrm{CMC}}$. Therefore, the $\sigma$ of the gel is substrate-sensitive in water and a low SDS concentration but becomes substrateinsensitive at an SDS concentration higher than $C_{\mathrm{CMC}}$. However, in the case of LASS and DBS, we 
always observed a higher friction on the silicon substrate than that on glass even at the highest surfactant concentration. This result indicates that the silicon substrate was not completely covered by these two surfactants.

As shown in Figure 3a, in SDS, $\sigma$ decreased dramatically around its $\mathrm{C}_{\mathrm{CMC}}$ on the hydrophobic Dglass substrates, while it began to decrease gradually from a concentration that was substantially lower than its $\mathrm{C}_{\mathrm{CMC}}$ on the hydrophilic glass. However, in LASS (Figure 3b), $\sigma$ began to decrease gradually from a concentration that was substantially lower than its $\mathrm{C}_{\mathrm{CMC}}$, both on hydrophilic glass and silicon wafer. On the other hand, in DBS (Figure 3c), $\sigma$ decreased dramatically around its $\mathrm{C}_{\mathrm{CMC}}$ on both substrates. These results suggest that the surfactant adsorption behavior is specifically related to the kinds of surfactant molecules and substrates, and is not necessary determined by the $\mathrm{C}_{\mathrm{CMC}}$. However, it is not understood why $\sigma$ increased slightly at a low concentration in the case of DBS.

We should emphasize that this dramatic friction reduction phenomenon with the addition of a surfactant only occurred for a soft and wet hydrogel on glass, not for friction between hard glass surfaces. As shown in Figure 2(c), the surfactant did not reduce $\sigma$ at any concentration and velocity. For glass vs. glass, the interfacial interaction is repulsive, the same as PNaAMPS gel vs. glass, due to the formation of electric double layer. However, the elastic modulus of a glass (approx. $10 \mathrm{GPa}$ [24]) is about five orders of magnitude higher than that of PNaAMPS gel. Comparing the results in Figure 1(a) (gel vs. glass) with those in Figure 2(c) (glass vs. glass), it is clear that surfactant can form film at the gel-glass interface, due to the soft nature of the gel.

\section{Discussions}

The behavior of gel friction in surfactant solution can be understood by the reduction of the boundary lubrication component. According to the physical picture described by Eq. 1, the surfaces of soft gels and hard solids are not ideally flat, but have asperities or misalignments on various spatial scales [19]. So the frictional stress $\sigma$ for PNaAMPS gel on a glass substrate is composed of: 1) the velocityindependent boundary lubrication term, $\sigma_{0}$, due to the direct contact between asperities, and 2) the velocity-dependent hydrodynamic lubrication term $\sigma_{\text {vis }}$, due to the existence of a liquid layer at other 
areas in addition to the asperities in contact. As shown in Figure 1, in water, the friction was less velocity-dependent, indicating that the boundary lubrication term dominated the friction over the entire velocity range. In a surfactant solution, the surfactant adsorbed at the interface of the asperities and reduced the magnitude of the first term of Eq. 1. As a result, the second term dominated the frictional behavior, and we observed a friction reduction at the low velocity region and a strong velocitydependency of friction. By supposing that the friction is only due to the second term in Eq. 1, we can estimate the average liquid layer thickness for the case in surfactant solution of high concentration using the relation $\sigma_{\mathrm{vis}}=\eta v / h$. At the highest velocity of $3.75 \times 10^{-3} \mathrm{~m} / \mathrm{s}$, the $h$-value is estimated as $10-$ $30 \mathrm{~nm}$ (in this order estimation, for simplification, we used the viscosity of water for $\eta$ ), which seems reasonable to be considered as a continuous lubricating layer. At the lowest velocity of $3.75 \times 10^{-6} \mathrm{~m} / \mathrm{s}$, however, the $h$-value is estimated to be $0.5-2 \mathrm{~nm}$. This value is too small to be treated as a liquid film, which suggests that some boundary lubrication, mediated by adsorbed surfactant, may still remain.

For the contact of nominally flat surfaces with random roughness, the local contact pressure is independent of the nominal $P$ but depends on the material elasticity and the topological feature of the surfaces [24-28]. For the friction between a soft gel and a hard solid, the local contact pressure is low due to the low elasticity of the gel. So surfactant molecules could remain at the asperities, as illustrated schematically in Figure 4(a). Many fundamental works have been done on the shear properties of selfassembled monolayers of surfactant coated on solid surfaces such as mica either in air or in aqueous conditions using the microscopic method such as surface force balance [29-34]. These studies have revealed that the friction is dramatically reduced by the presence of surfactant at the interface. We assume that similar friction reduction effect occurred in the case of gel-solid interface due to the adsorption of the surfactant.

For the friction between hard solids, like glass vs. glass, the local contact pressure at the asperities becomes too high for surfactant molecules around such highly pressed points to form the adsorbing layer. As a result, the asperities contact directly without the intervention of the surfactant layer, as illustrated schematically in Figure 4(b). This explains why no friction reduction phenomenon was observed for glass-glass friction in the surfactant solutions. 
It should be noted that surfactants are well-known lubricant molecules frequently used to reduce friction between soft solids such as rubbers [35-37]. According to the study on rubber friction, semipermanent thin film layers of 10-15 nm exist for static contacts of rubber on glass over a pressure range 10-60 $\mathrm{kPa}$, in the presence of sodium dodecyl sulfate (SDS), an ionic surfactant, aqueous solution [37]. The presence of these apparent equilibrium films was attributed to electric double layer repulsion that arises from the adsorption of SDS to rubber and glass from the aqueous medium. The films collapse at contact pressures higher than $60 \mathrm{kPa}$. The strength of the film against the contact pressure may be due to hydrophobic interaction between SDS molecules.

The present results of hydrogel are similar to these previous studies on rubbers. However, since the hydrogel consists of crosslinked polymer network with a mesh size of several $\mathrm{nm}$ that is comparable or larger than the surfactant molecules, the detailed molecular dynamics of the surfactant in the present case might be quite different from that confined between two solid surfaces. It is suggested that when $C_{\text {surf }}$ is higher than a critical concentration, $C_{\mathrm{t}}$, a continuous surfactant layer is formed at the interface of the asperities between the gel and hard substrate, which might have two effects: 1) inducing charge at the interface, 2) increasing the contact area and reducing the local contact pressure. Effect 1) will induce the formation of the electric double layer since both of the solid substrate coated with surfactant molecules and the hydrogel surface are negatively-charged. Effect 2) will increase the thickness $h$ of the electric double layer at the interface since $h$ is related to the normal pressure as $h \propto P^{-1 / 2}$ [38]. When the gel is in sliding, the electric double layer serves as the lubrication layer and thus to reduce the friction, which is similar to the friction scenario of the charged polymer brushes under shear [39].

\section{Conclusions}

Surfactants can significantly reduce the friction of soft and wet interfaces sliding at a moderate velocity, for example, the friction of a soft gel vs. hard solid, the same as that observed for rubber friction. This friction reduction effect by a surfactant disappears for friction between hard interfaces, for example, the friction of glass $v s$. glass. The surfactant concentration needed to form the "screening layer" of surfactant, $C_{\mathrm{t}}$, is not necessarily in exact accordance with the $C_{\mathrm{CMC}}$ value, but depends on the 
surfactant-substrate interaction. For SDS, when the surfactant concentration is higher than several times the value of $C_{\mathrm{t}}$, the frictional stress $\sigma$ is no longer dependent on the hydrophilic/hydrophobic degree of the substrates, probably due to formation of a continuous layer of surfactant at the interface. In daily life, the formation of such a screening layer of surfactant on a soft and wet surface is responsible for the improved smooth feeling of skin with the application of a cosmetic soap, cream, or shampoo.

Acknowledgments. We thank Prof. K. Tsujii, Nano-devices Laboratory, Nanotechnology Research Center, Research Institute for Electronic Science, Hokkaido University, for the valuable advice. This research was financially supported by a Grant-in-Aid for Specially Promoted Research (No. 18002002) from the Ministry of Education, Science, Sports and Culture of Japan. 


\section{Figure and Table Captions}

Figure 1. Velocity dependence of the frictional stress for a PNaAMPS gel disk sliding on hydrophilic

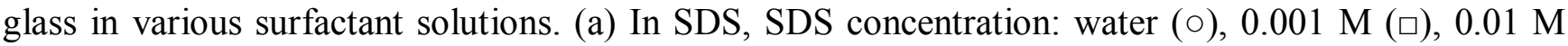
$(\diamond)$, and $0.1 \mathrm{M}(\Delta)$; (b) In LASS, LASS concentration: water $(\circ), 0.001 \mathrm{M}(\square), 0.01 \mathrm{M}(\diamond)$, and $0.1 \mathrm{M}$ $(\Delta)$; (c) In DBS, DBS concentration: water $(\circ), 0.0001 \mathrm{M}(\square), 0.001 \mathrm{M}(\diamond)$, and 0.01 M ( $\Delta)$. Load: 0.4 $\mathrm{N}$, nominal pressure: $2.2 \mathrm{kPa}$, gel thickness: $2.75 \mathrm{~mm}$. In (a), $(>)$ denotes the results in a $0.1 \mathrm{M} \mathrm{NaCl}$ solution.

Figure 2. Velocity dependence of the frictional stress for a PNaAMPS gel sliding on hard solids with various levels of hydrophobicity in an SDS surfactant solution. (a) PNaAMPS gel vs. silicon wafer; (b) PNaAMPS gel vs. D-glass. Sliding velocity dependence of the frictional stress for glass vs. glass is shown in (c). SDS concentration: water $(\circ), 0.001 \mathrm{M}(\square), 0.01 \mathrm{M}(\diamond), 0.1 \mathrm{M}(\Delta)$, and $0.3 \mathrm{M}(\nabla)$.

Figure 3. Surfactant concentration dependence of the frictional stress for a PNaAMPS gel sliding on hydrophilic glass (०), silicon wafer $(\square)$, and hydrophobic D-glass $(\diamond)$. (a) In SDS, (b) LASS, and (c) DBS. Sliding velocity: $3.75 \times 10^{-6} \mathrm{~m} / \mathrm{s}$.

Figure 4. Schematic illustration for the effect of material softness on the contact in the presence of a surfactant. The mean contact pressure at the asperities is not dependent on the nominal pressure $\mathrm{P}$ but depends on the elasticity and surface morphology of the materials in contact. (a) For gel vs. glass, the local pressure at the asperities in contact is low (with an order of magnitude of $1 \mathrm{MPa}$ ), due to the softness of the PNaAMPS gel. Surfactant molecules can absorb at the interface of the asperities, and serves as the lubricant to reduce the friction. (b) For glass vs. glass, the asperities are in contact with a high local pressure (with an order of magnitude of $10^{4} \mathrm{MPa}$ ), due to the rigidity of glass. Under such a high local pressure, the surfactant molecules cannot form a continuous adsorbing layer between the asperities in contact, and does not reduce the friction.

Scheme 1. Chemical structures of the various surfactant molecules used in this study. 


\section{References}

(1) de Gennes, P. G. Scaling Concept in Polymer Physics; Cornell University Press: Ithaca, NY, 1979.

(2) Gong, J. P.; Soft Matter, 2006, 2, 544-552.

(3) Gong, J. P.; Iwasaki, Y.; Osada, Y.; Kurihara, K.; Hamai, Y. J. Phys. Chem. B 1999, 103, 6001.

(4) Gong, J. P.; Kurokawa, T.; Narita, T.; Kagata, G.; Nishimura, G.; Kinjo, M.; Osada, Y. J. Am. Chem. Soc., 2001, 123, 5582.

(5) Kagata, G.; Gong, J. P.; Osada, Y. J. Phys. Chem. B 2002, 106, 4596.

(6) Baumberger, T.; Caroli, C.; Ronsin, O. Phys. Rev. Lett. 2002, 88, 75509.

(7) Baumberger, T.; Caroli, C.; Ronsin, O. Eur. Phys. J. E 2003, 11, 85.

(8) Nitta, T.; Kato, H.; Haga, H.; Nemoto, K.; Kawabata, K. J. Phys. Soc. Jpn. 2005, 74, 2875.

(9) Tominaga, T.; Takedomi, N.; Biederman, H.; Furukawa, H.; Osada, Y.; Gong, J. P. Soft Matter, 2008, 4, 1033.

(10) Yamaguchi, T.; Ohmata, S.; Doi M. Journal of Physics: Condensed Matter, 2009, 21, 205105.

(11) Freeman, M. E.; Furey M. J.; Love, Brian J.; Hampton, Jeanne M.; Wear, 2000, 241, 129-135.

(12) Kim, S. H.; Opdahla, A.; Marmob, C.; Somorjai, G. A. Biomaterials, 2002, 23, 1657.

(13) Pan, Y-S.; Xiong, D-S.; Chen, X-L.; J. Tribol.; 2008, 130, 031802.

(14) Morita, Y.; Tomita, N.; Aoki, H.; Sonobe, M.; Wakitani, S.; Tamada, Y.; Suguro, T.; Ikeuchi, K. J. of Biomechanics, 2009, 39, 103-109.

(15) Haraguchi, K.; Takada, T.; Macromolecular Chemistry and Physics, 2005, 206, 1530-1540.

(16) Buschmann, M. D.; Grodzinsky, A. J. J. Biomech. Eng. 1995, 117, 179.

(17) Fung, Y. C. Biomechanics: Mechanical Properties of LiVing Tissues, 2nd ed.; Springer-Verlag: New York, 1993.

(18) Lee, S. H. and Spencer, N. D, Science, 2008, 319, 575.

(19) Oogaki, S.; Kagata, G.; Kurokawa, T.; Osada, Y.; Gong, J. P. Soft Matter, 2009, 5, 1879. 
(20) Hills, B. A.; Butler, B. D.; Barrow, R. E.; J Appl. Physiol., 1982, 53: 463-469.

(21) Butler, (B. D.; Lichtenberger, L. M.; Hills, B. A.; Am. J Physiol. Gastrointest. Liver Physiol. 1983, 244: G645-G651.

(22) Hills, B. A.; Burke, J. R.; Thomas, K; Perit. Dial Int. 1998, 18(2): 157-165.

(23) Kurokawa, K.; Gong, J. P., Osada, Y.; Macromolecules,2002, 35(21), 8161-8166.

(24) Davidege, R. W.; Green, T. D. J. Mat. Sci. 1968, 629.

(25) Johnson, K. L. 1966, Contact mechanics, Cambridge: Cambridge University Press.

(26) Greenwood, J. A.; Williamson, J. B. P. Proc. R. Soc. 1966, A295, 300.

(27) Persson, B. N. J. Phys.: Condens. Matter, 2005, 17, R1.

(28) de Gennes, P. G.; Brochard-Wyart, F.; Quere, D. Capillarity and Wetting Phenomena: Drops, Bubbles, Pearls, Waves; Springer, New York, 2003.

(29) Yamada, S.; Israelachvili, J. N. - J. Phys. Chem. B, 1998, 102, 234.

(30) Pashley, R. M.; Israelachvili, J. N. - Colloids and surfaces, 1981, 2, 169.

(31) Meyer, E. E.; Lin, Q.; Hassenkam, T.; Oroudjev E.; Israelachvili, J. N.; PNAS 2005, 102, 68396842.

(32) Briscoe, W. H.; Klein, J. J. of Adhesion, 2007, 83:705-722.

(33) Vakarelski, I. U.; Brown, S. C.; Rabinovich, Y. I.; Moudgil, B. M.; Langmuir 2004, 20, 17241731.

(34) Liu, Y.; Evans, D. F.; Song, Q.; Grainger, D. W.; Langmuir 1996, 12, 1235-1244

(35) Richards S. C.; Roberts A. D.; J. Phys. D: Appl. Phys., 1992,25, A76-A80.

(36) Hardy, W. B. Collected Works, 1936, Camb. Univ. Press.

(37) Gregory, J. N. C.S.I.R. (Australia) Tribophysic Division Report A, 1943, 74.

(38) Gong, J. P.; Kagata, G.; Osada, Y. J. Phys. Chem. B 1999, 103, 6007.

(39) Raviv, U.; Giasson,S.; Kampf, N.; Gohy, J.-F.; Jerome, R.; Klein, J.; Nature, 2003, 425, 163. 


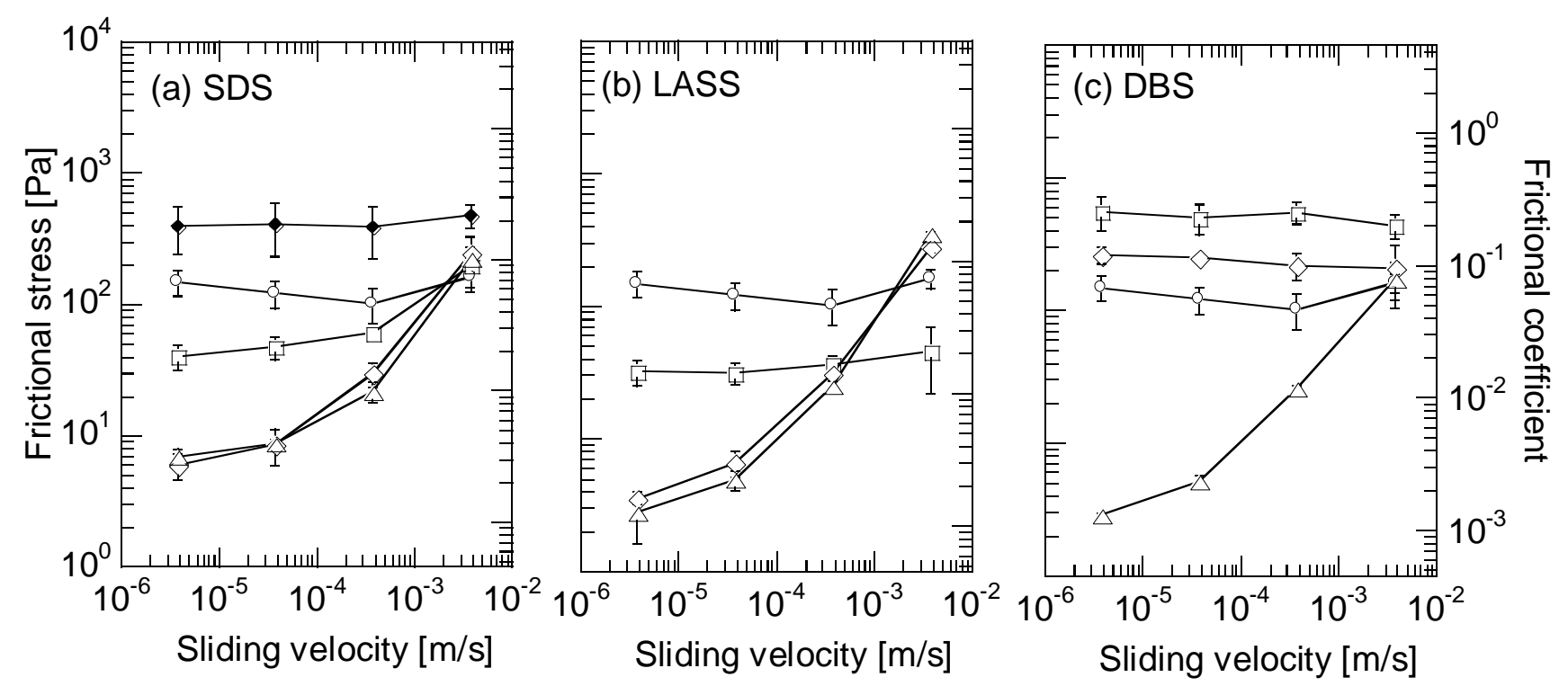

Figure 1. K. Kamada, et al. 

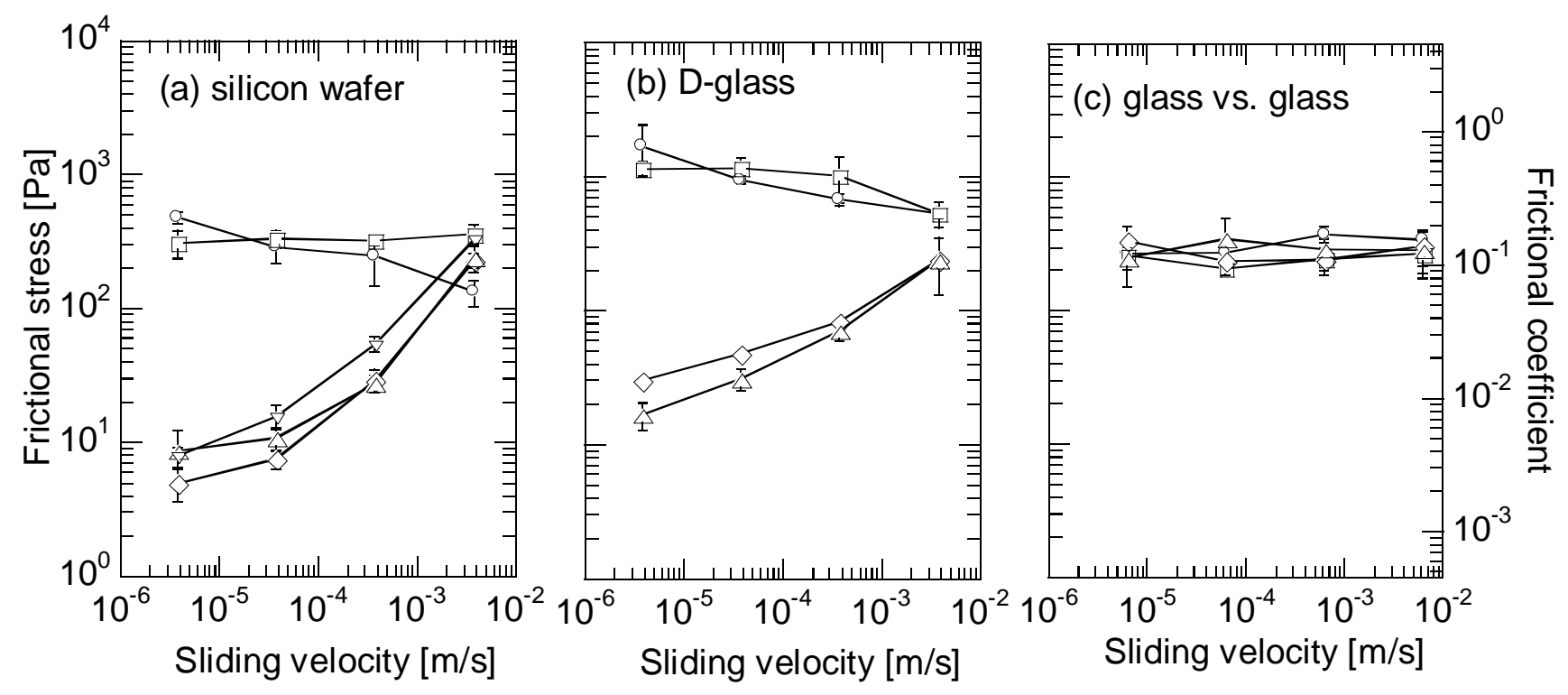

Figure 2. K. Kamada, et al. 


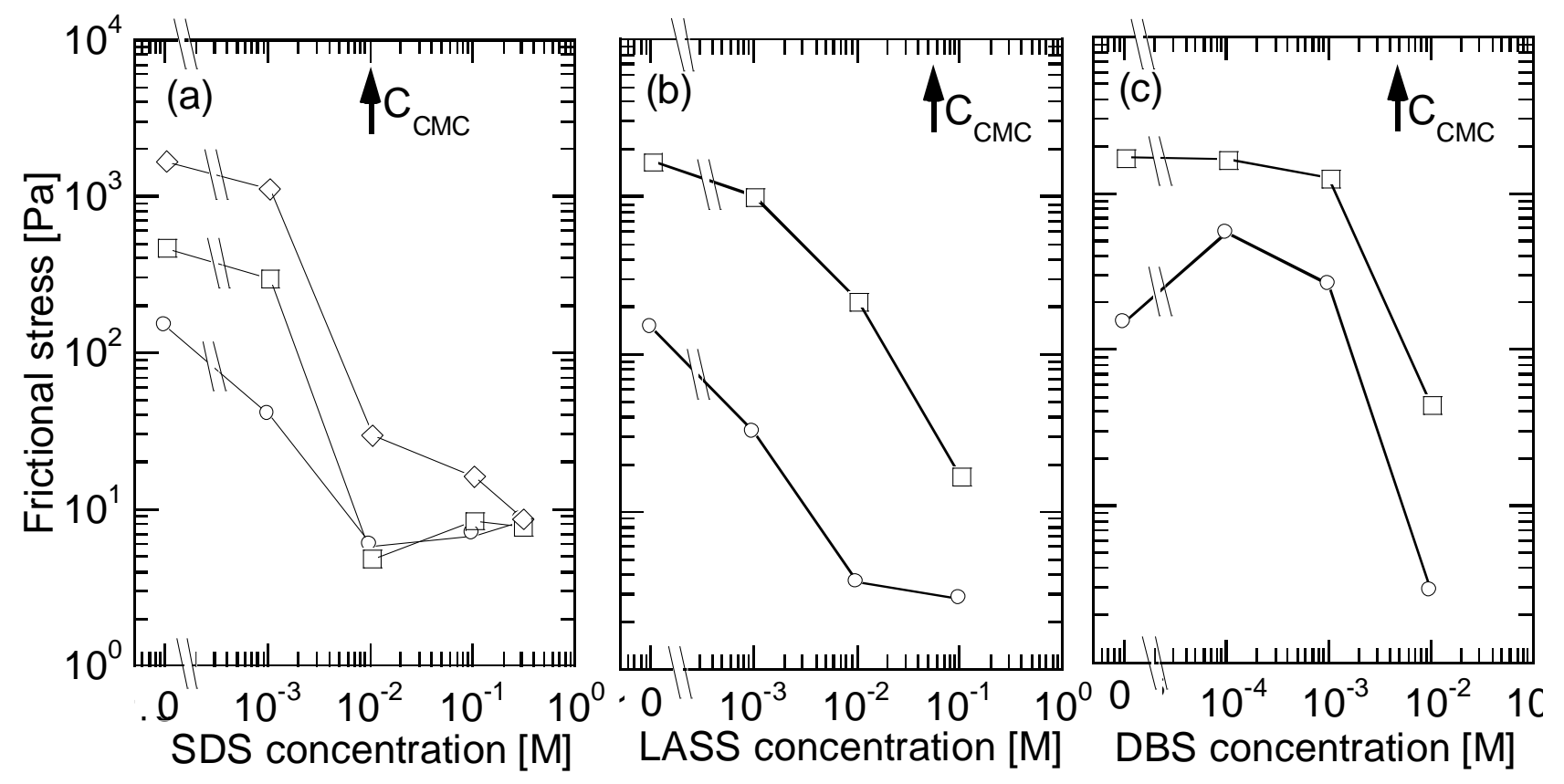

Figure 3. K. Kamada, et al. 
(a)
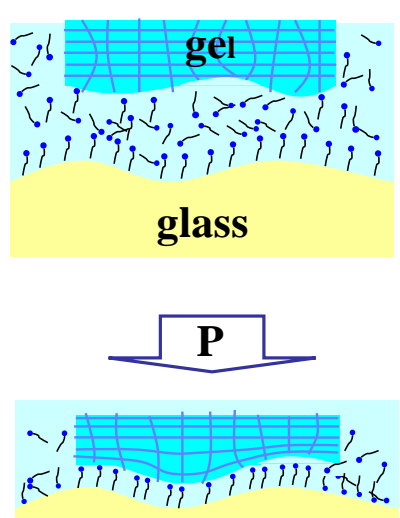

(b)
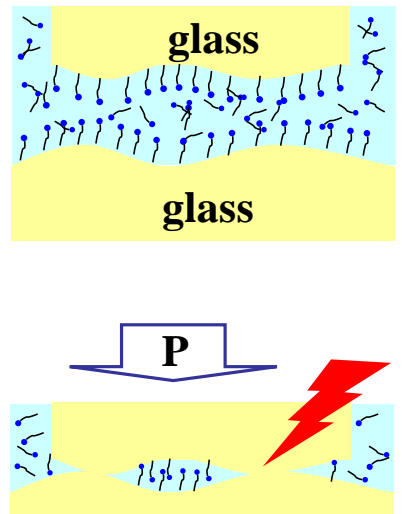

Figure 4. K. Kamada, et al 
Scheme 1. Chemical structures of the various surfactant molecules used in this study.

\begin{tabular}{|c|c|c|}
\hline Name & \multicolumn{1}{|c|}{ Structure } & $\mathrm{CMC}$ \\
\hline $\begin{array}{c}\text { Sodium dodecyl sulfate } \\
\text { (SDS) } \\
\begin{array}{c}\text { Dodecylbenzene } \\
\text { sulfonic acid, } \\
\text { sodium salt } \\
\text { (DBS) }\end{array}\end{array} \mathrm{CH}_{3}\left(\mathrm{CH}_{2}\right)_{11} \mathrm{OSO}_{3} \mathrm{Na}$ & $0.008 \mathrm{M}$ \\
\hline $\begin{array}{c}\text { Lauric acid, sodium salt } \\
\text { (LASS) }\end{array}$ & $\mathrm{CH}_{3}\left(\mathrm{CH}_{2}\right)_{11}$ & $0.0014 \mathrm{M}$ \\
\hline
\end{tabular}


TOP graphic

(a)
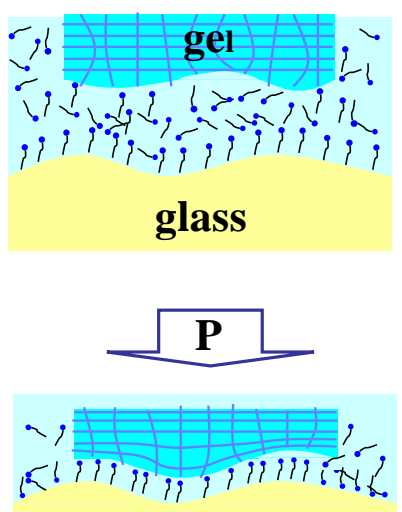

(b)
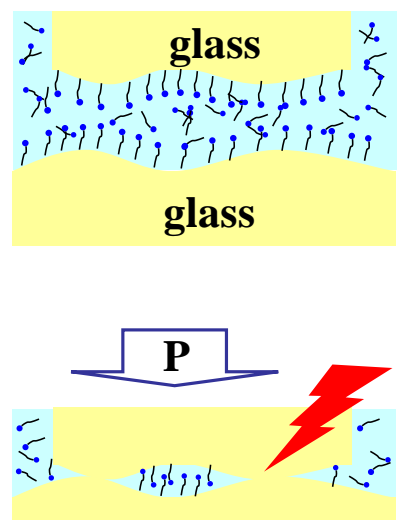

A dramatic reduction in the surface friction of gel was detected by the addition of a surfactant to the water medium. Reduction occurred at gel-solid friction (a) but not at solid-solid friction (b), indicating that the softness of the gel surface was crucial for this surfactant-induced friction reduction. 